\title{
The RoadRobot project: from theory to practice in systems integration
}

\author{
J.P.Pimentão ${ }^{\star+} ;$ R.Gonçalves $^{+} ;$T.Gonçalves $^{+} ;$H.Silva $^{+} ;$M.Vital $^{\dagger}$; \\ P.Sousa ${ }^{\dagger} ;$ A.Garçãoo $o^{\star+}$ \\ *New University of Lisbon - Fac. Sciences and Technology; \\ +Uninova - Center for Intelligent Robotics \\ Quinta da Torre, 2825 - Monte Caparica, Portugal \\ Tel/Fax+351-2-3500200/2941253 E-mail:pim,rg@uninova.pt
}

\begin{abstract}
This paper presents the experience of Uninova on the European Commission's (EC) ESPRIT III project 6660 - RoadRobot - Operator Assisted Mobile Road Robot for Heavy Duty Civil Engineering Applications.

The objective of RoadRobot (currently on its fourth and last year) is the development of a generic multipurpose control architecture for integrated outdoor building sites composed by mobile heavy-duty platforms. This architecture is demonstrated by applying it to road building. The experience on the definition of the hardware and software architecture capable of supporting this type of applications is presented and the data modelling, supported by ISO 10303 - STEP, is then summarised.

Some focus is put on the Uninova's STEP-based Integration Platform, which is the tool used to facilitate the task of integrating the RoadRobot's heterogeneous software packages.

The paper concludes with a brief presentation of the relevant results of the project.
\end{abstract}

\section{Keywords}

Modelling, Integration, Heterogeneous Systems, Standards, Industrial experiences

\section{BACKGROUND}

The purpose of the EC's ESPRIT III - RoadRobot Project is the development of a generic and flexible control architecture for integrated outdoor building sites (Pimentão, 1993). This architecture should be applicable to construction sites with mobile heavy-duty equipment, from road building to dam or airport construction. The project's demonstration (by the end of April of 1996) will be on road building.

To this end, a project consortium was formed, composed of seven European partners ranging from $R \& D$ institutes to software houses, and including a road paver industry. 
Keeping in mind the practical example of road construction, RoadRobot's aim was to produce an architecture that might be able to control a fully automated construction site. The input is the data usually fed to construction companies (project information coming from Computer Aided Engineering - CAE - systems) and, in the end, the architecture enables the control of a set of machines performing the construction work.

The project started with a one year definition phase, where the RoadRobot's architecture was defined and the major modules and interfaces identified.

At the end of that year the consortium decided to use of ISO 10303 - STEP (Standard for the Exchange of Product model data) as modelling tool (ISO-1, 1992). STEP was therefore used for modelling all data interchange among the modules at the various layers of the RoadRobot's architecture. This includes the modelling of all data exchanged between modules, as well as the definition of all the messages.

The following two years were devoted to the implementation of the defined modules, and the last year is being used for the integration of the several modules that will culminate on the final demonstration.

Besides the definition of the generic architecture, some problems had to be tackled in order to enable a demonstration under real construction conditions.

One of the problems that had to be dealt was the production of a navigation system (by UK Robotics) for use on excavators, pavers, etc. The navigation module produced is capable of supplying the vehicle's position (with an accuracy of $2 \mathrm{~cm}$ ) and orientation.

The other problem still being tackled is the creation of communication links to the machines. The consortium's solution is to use TCP/IP in all interfaces between computers. This means that the machines' interface also uses TCP/IP. The problem, however, is that in this interface (due to the mobile characteristics of the machines) an ethernet cable is not a possible solution. Several possibilities (from wireless RS232 links to wireless networks) are being studied, but due to the fact that the communicating machines may be several kilometres apart, the solution is not yet found.

\section{THE ROADROBOT ARCHITECTURE}

After several meetings with people from construction companies, and a set of consortium meetings, a four-layer architecture was produced (Figure 1): Site, Cell, Machine and Tool (Pimentão, 1993 and 1994).

In general terms, a Site is responsible for a set of Cells; a Cell is responsible for a set of machines and a machine has a set of Tools.

Project data is fed from the CAE tool into the Site Controller where planning and scheduling activities take place. These activities consider the set of Resources available at the Site, and group them into working cells (each cell being of a given cell type and capable of performing a set of related tasks, for example excavating cells, paving cells, etc.).

At the cell level a similar process takes place, i.e. taking into account the set of resources available and the cell's assigned task, the task is processed via planning and scheduling tools producing the set of activities for each of the machines.

In due time, information is passed from a higher layer of the architecture to a lower one. Each controller monitors and controls every lower level controller in the performance of its assigned tasks.

A controller may, therefore be seen as a system capable of performing a set of functions that range from task decomposition, planning and scheduling, to control and monitoring of a set of 
lower level controllers.

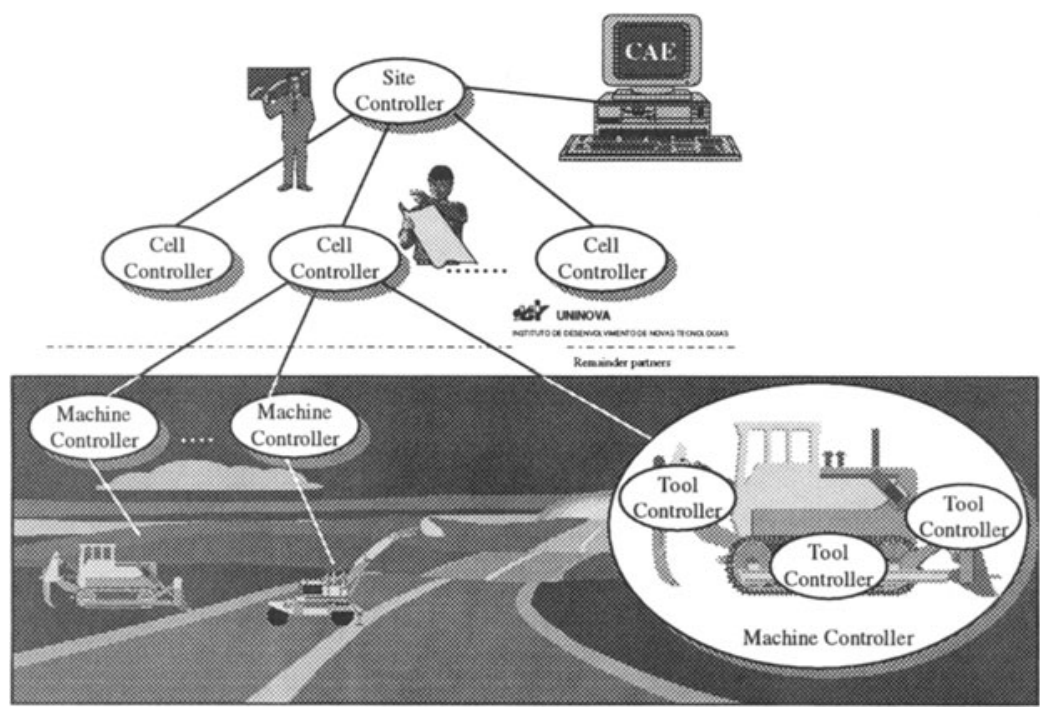

Figure 1 RoadRobot's Architecture.

In general each controller may have three interfaces (Figure 2): (1) the interface to the upper level controller, (2) the interfaces to the lower level controllers and (3) an interface for the controller's operator (the entity that allows the control and monitoring of the lower level controllers, in the absence of upper level controllers).

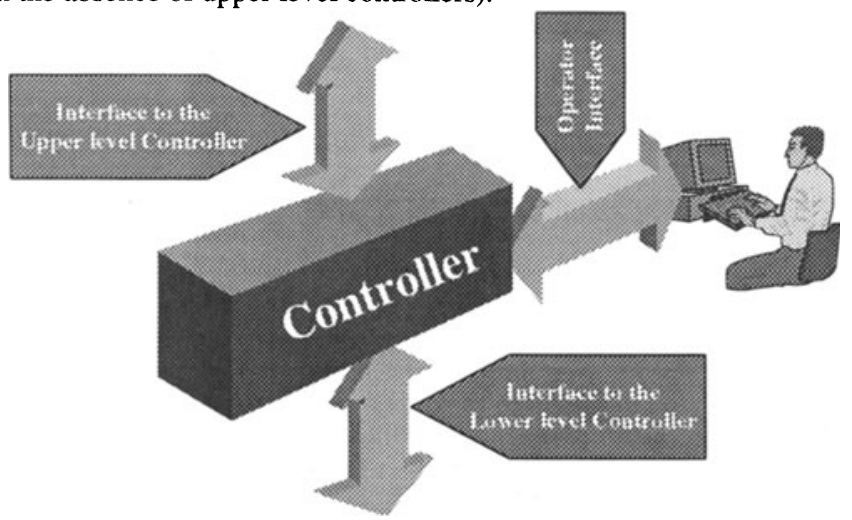

Figure 2 - A controller and its interfaces.

Some of the constraints presented by the Project specification were the efficient management of resources and efficient planning and scheduling. It was clear to the consortium that, in one hand, there might be a set of existing software tools (legacy systems) that could perform efficiently the tasks required at the controller and, on the other hand, that it was not 
RoadRobot's purpose to develop the needed tools. In the case of road construction, the scenario proved to be quite the opposite, and some tools (planners and schedulers) had to be developed, in order to demonstrate the architecture's functionality.

Nevertheless, since the purpose of the project is to produce a flexible architecture, the system was developed assuming the existence of legacy systems (which may be true in other applications).

The consortium selected a standard way (STEP) for the exchange of information among modules. This became also true for the exchange of information among applications at a given controller (Site or Cell). Each controller's architecture is based on the existence of an Information System that facilitates the exchange of information between applications. This task is supported by Uninova's STEP-based Integration Platform (SIP) that is later described on point 4. of this document.

\section{THE DATA}

This point is devoted to the summary presentation of the EXPRESS schemata used by RoadRobot to represent the information flows within the architecture.

There are two types of information flows: the ones that occur through a given interface and the ones that occur between applications within a controller.

The former flows can then be split into three categories: Commands, Tasks and Responses to the previous categories. The later flows depend on the specific application at hand.

It is clear that Tasks are application dependent. However, Commands and Responses are further divided into application independent and application dependent. Examples of Application Independent Commands are: Query Cell Status, Fast Stop, etc.; Application Dependent Commands and Responses can be: Foresee lack of asphalt, Orientation unobtainable, etc.

Regarding the specific application (road paving), road construction data had to be modelled. In fact, one of the first modelling tasks was the definition of a model for representing data imported from the selected CAE tool (InRoads from Intergraph). The model produced by Uninova is based on a previous one - the Road Model Kernel (RMK) - produced by TNO and the Dutch Ministry of Public Works (Willems, 1990). RMK was the result of a modelling effort made for a similar CAE tool (MOSS from MOSS Systems Ltd).

The model produced by Uninova extends RMK with geologic information (for excavating tasks) and road layer information (for paving tasks).

Other data models were produced for representing data at Site and Cell level, such as resource representation, planning and scheduling data.

The Express models produced for the RoadRobot project are available at the WWW page of the RoadRobot project: http://www.uninova.pt/CRI/GR_SSNC/RESEARCH/roadrobot.html.

\section{THE TOOL: THE STEP-BASED INTEGRATION PLATFORM - SIP TOOLKIT}

To provide a set of mechanisms that speed up the integrator's tasks, facilitating the generation of interfaces and avoiding development errors, becomes essential if one wants to achieve integration results in a competitive time. Because company's needs are constantly changing, to spend a large amount of time to fully integrate a company is unacceptable (Helpenstein 1991).

The use of standards in the modelling processes can avoid the development of a high 
number of interfaces and extend the life time of the resultant system. In time, as the commercial tool's builders absorb these standards, the effort needed to keep the global system updated will be reduced.

To assist information exchange among tools, UNINOVA developed a STEP-based Integration Platform - SIP toolkit (Jardim-Gonçalves, 1994). SIP is a set of tools, developed in the framework of the European BRITE/EURAM CIMTOFI (CIM sysTems with improved capabilities fOr Furniture Industry) project (Jardim-Gonçalves, 1993), that support the integration of several applications using a unique neutral data format (ISO 10303) (ISO-1, 1992). The goals are reached by adapting existing applications selected in the market, and integrating product models, processes and resources in a global and standard information system.

SIP's main requirements were: (1) Support the integration (data exchange) of all existing and future activity tools, which are heterogeneous by nature; (2) Offer the possibility of product data exchange between different sites; (3) Provide a good approach for data modelling at all levels, preferentially using a standard; (4) Enable the management of expertise knowledge related to specific activities, using a standard interface; (5) Allow interfacing to different standard languages (e.g. C, C++, EIFFEL, Object Oriented Data Bases and Relational Data Bases).

\subsection{SIP's Architecture}

SIP is fundamentally constituted by an Information Management System (IMS), an Information System Access Protocol (ISAP) and a Development System Tool Set (DSTS). The architecture of SIP is presented on Figure 3.

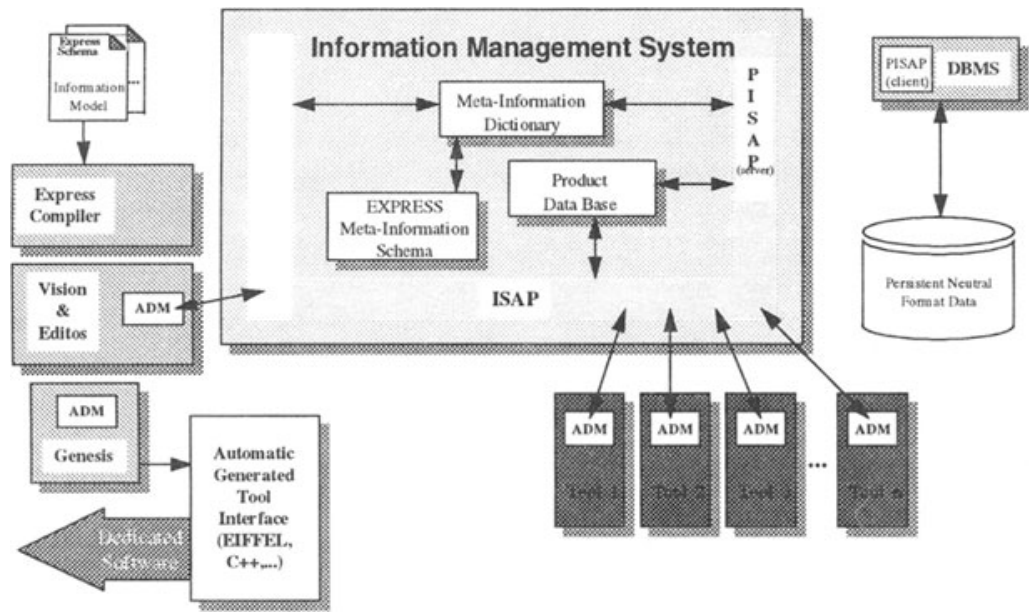

Figure 3 SIP's architecture.

The IMS handles the information based on a model (EXPRESS schema), and it is constituted by:

- PISAP - Persistence Driver; 
- MID - Meta-Information Dictionary;

- PDB - Product Data Base;

- EXPRESS MIS - EXPRESS Meta-Information Schema;

- ISAP - communication protocol. (Interface between external tools and IMS, allowing data exchange in a distributed way).

The architecture incorporates many of the concepts of Computer Integrated Manufacturing (CIM) to bring improvements in: productivity, flexibility and quality.

In order to allow a gradual integration of automated functions as they become available, a suitable object-oriented approach was adopted in the design of the architecture.

\subsubsection{SIP's Development System Tool Set components}

The SIP's Development System Tool Set (DSTS) components are:

- An EXPRESS compiler (ISO, 1991) (modelled itself in EXPRESS), that has the responsibility of processing Express schemata, checking for lexical, syntactic and semantic errors, generating the equivalent meta-information representation structure, and updating the meta-information dictionary inside SIP.

- Vision and Editus, that allow edition and navigation through the STEP-based Information System.

- Genesis, a tool that automatically generates the front end for several programming languages. Every time it is necessary to integrate a new tool, a dedicated Application Dependent Module (ADM) has to be developed. The DSTS Genesis helps this task, generating code automatically. This code stands for a complete implementation of all entities described in Express schemata, generating automatically access primitives for those entities (for example those that make the data transference from/to SIP using ISAP). Front ends to $\mathrm{C}, \mathrm{C}++$ and ONTOS (Object oriented data base) are already available and Relational Databases access languages are being produced (SQL is now in test).

- Import/Export data tool. This tool uses neutral format (Part 21) (ISO-2, 1992) to exchange data.

In order to be able to maintain flexibility, SIP runs on several platforms (IBM PC compatible, IBM RISC 6000, SUN workstations), and uses TCP/IP as the communication protocol.

\subsection{How to integrate tools using SIP}

When we want to integrate tools in a system, several steps should be followed in order to conduct the integration tasks. To integrate a tool using SIP, one has to: (1) Study the tool to be integrated in industrial system context, to understand which data must be imported/exported; (2) Model these data using ISO 10303/11 EXPRESS; (3) Compile the EXPRESS model using SIP's EXPRESS compiler; (4) Generate the Application Dependent Module (ADM) for the tool (ADM will be the piece of software that establishes the connection between the tool and the remainder system); (5) Integrate the generated ADM interface with the tool.

A detailed data analysis of each tool should be performed first, finding which data must be shared by system's tools. After the conclusion of this work, a consolidated Schema (described in EXPRESS) must represent the data structure of the global transfer necessities among system tools. Hence, the import/export data flowing through the system is defined, and it will be 
physically represented using the STEP neutral format, supported by the Express schema to impose the rules of data transference.

\subsection{New features of SIP regarding RoadRobot requirements}

The usage of SIP in the RoadRobot project raised new problems that could not be easily resolved using SIP's last version. Therefore, SIP is being improved and the following features are being developed:

- Automatic code generation, based on a dedicated language, regarding data transfer among tools modelled using different EXPRESS-based Application Protocols (AP) (ISO-1, 1992);

- E-Mail Data Interchange (EMDI), using neutral format (ISO 10303-21) to allow data transfer world-wide (for instance data exchange between the headquarters in Europe and the sub-offices world-spread);

- A mechanism that allows message flowing among tools inside the system. This mechanism will allow signalling of events in the Information System.

\section{THE RESULTS}

The RoadRobot Project deals with a segment of industry where very little effort has been put towards automation. Beyond hard and hazardous work, civil construction workers have to endure the roughness of the climate. Construction works are sometimes over-rated due not only to the massive volumes of man-power that they require, but also due to inefficient planning and scheduling of activities.

This project has provided a flexible platform for the production of semi-automated construction sites. At this point of development, it would be imprudent to assume that one could have a fully automated site; many security aspects have still to be analysed.

The RoadRobot architecture provides the means for development of such systems. The effort put in the demonstration of the project has had two major effects:

- It has demonstrated the functionality of RoadRobot's architecture and,

- It allowed the creation of a set of models that may be used in similar systems. It is the intention of the consortium to move towards the creation of an ISO Application Protocol for the road construction Industry, based on the results of this project.

In order to be able to demonstrate the architecture, specific planning and scheduling tools had to be developed for Road Construction. The comments received so far from the industry are very encouraging and it is Uninova's intention to continue the development of those applications.

Another side effect was the increase in the development of the STEP-based Integration Platform. SIP is currently being used on several Portuguese and European projects, covering the areas of Civil engineering, Furniture building, Moulding and Metal-mechanic industry.

It is the objective of Uninova to transform SIP into a commercially available tool. Some contacts have already been established for this purpose.

\section{REFERENCES}

Camarinha-Matos, L. M. and Osório, L. (1992) CIM information Management System: An 
Express-Based Integration Platform. In Proceedings of IFAC - Workshop on CIM in Process and Manufacturing Industry, 23-25 Nov 92, Espoo, Finland.

Helpenstein, H. (1991) CAD Geometry Data Exchange Using STEP.Springer-Verlag.

ISO-1 TC184/SC4/WG7 N262 (1992) ISO 10303 - Part1 - Overview and Fundamentals Principles.

ISO TC184/SC4/WG5 (1991) ISO 10303 - Part11 - EXPRESS Language Reference Manual. ISO-2 TC184/SC4/WG7 N262(1992)ISO10303-Part21-Clear text encoding of the Exchange Structure.

Jardim-Gonçalves, R.; Martins-Barata, M. and Steiger-Garção, A. (1993) CIMTOFI project Deliverables and reports.

Jardim-Gonçalves, R.; Sousa, P.; Vital, M.; Silva, H. and Steiger-Garção, A. (1994) SIP Reference and User Manual. Uninova, Portugal.

Jardim-Gonçalves, R.; Sousa; Vital, M.; Silva, H. and Steiger-Garção, A. (1995) ATECNIC project - Deliverables and reports. Uninova. Portugal.

Jardim-Gonçalves, $\mathrm{R}$ et. al. (1995) Integrating applications for the construction industry using SIP. In Proceedings of CIB W78, University of Stanford - Palo Alto - California - USA.

Pimentão, J.P. et al. (1993) Architecture of the Site Controller for Road Building Applications". In P.A.MacConaill C.Kooij and J.Bastos, editors, Realizing CIM's Industrial Potential, pages 358-367. IOS Press, Amsterdam, May 1993.

Pimentao, J.P.; Azinhal, R.; Goncalves, T.; and Steiger-Garção, A. (1994) RoadRobot project - Deliverables and reports. Uninova, Portugal.

Rembold, U. and Dilman, R. (1986) Computer-Aided Design and Manufacturing. SpringerVerlag.

Willems, P. (1990) The Road Model Kernel. Technical Report TNO-B-89-831(E), TNO Building and Construction Research, Jan 90

\section{BIOGRAPHY}

João Paulo Pimentão, Assistant at the Dep. of Elec. Engineering of the FCT/UNL and Researcher at Uninova; Computer Engineering degree (CED) and MSc (1994) in Computer Engineering by FCT/UNL.

Ricardo Jardim Gonçalves, Researcher at Uninova; BSc in Electronics and Telecommunications at the Polytechnic Institute of Lisbon, CED by FCT/UNL. Currently pursuing MSc in Operative Research and System Engineering at the Technical Univ. of Lisbon.

Teresa Gonçalves, Researcher at Uninova; CED by FCT/UNL, currently pursuing MSc in Computer Engineering by FCT/UNL

Helder Silva, Researcher at Uninova; BSc in Electronics and Telecommunications by the Polytechnic Institute of Lisbon, is concluding his degree in Systems and Telecommunications at the same school.

Miguel Vital and Pedro Sousa, Researchers at Uninova; BSc in Electronics and Telecommunications by the Polytechnic Institute of Lisbon; have recently concluded their CED by FCT/UNL.

Adolfo Steiger Garção, Full professor at the Dep. of Elec. Engineering of the FCT/UNL, President of Uninova and Director of Uninova's Intelligent Robotics Centre. Responsible for several international research projects and over 100 National and international publications. 